\title{
THE MCKAY-THOMPSON SERIES ASSOCIATED WITH THE IRREDUCIBLE CHARACTERS OF THE MONSTER
}

\author{
KOICHIRO HARADA AND MONG LUNG LANG
}

\begin{abstract}
Let $\mathbb{V}=\coprod_{\bar{\sim}=\nvdash}^{\infty} \mathbb{V}_{\bar{\sim}}$ be the graded monster module of the monster simple group $\mathbb{M}$ and let $\chi_{k}$ be an irreducible representation of $\mathbb{M}$. The generating function of $c_{h k}$ (the multiplicity of $\chi_{k}$ in $\mathbb{V}_{\bar{\sim}}$ ) is determined. Furthermore, the invariance group of the modular function associated with the generating function is also determined in this paper.
\end{abstract}

\section{INTRODUCTION}

Let $\mathbb{M}$ be the monster simple group and $\mathbb{V}$ be the monster module of FrenkelLepowsky-Meurman [3]. $\mathbb{V}$ is a graded $\mathbb{M}$ module

$$
\mathbb{V}=\coprod_{\bar{\sim}=\nvdash}^{\infty} \mathbb{V}_{\bar{\sim}}
$$

such that

$$
j(q)-744=\sum_{h=0}^{\infty} \operatorname{dim} \mathbb{V}_{\bar{\sim} \|} \bar{\sim}^{-\nVdash} .
$$

In particular, $\operatorname{dim} \mathbb{V}_{\nvdash}=\nVdash, \operatorname{dim} \mathbb{V}_{\nVdash}=\nvdash, \operatorname{dim} \mathbb{V}_{\not}=\nVdash \nrightarrow 丸 \nleftarrow \nleftarrow \not, \operatorname{dim} \mathbb{V}_{\nVdash}=\not \nVdash \nVdash \nsupseteq \nrightarrow \nVdash \triangleright \Varangle \nvdash$, $\cdots$. Let $\chi_{k}, 1 \leq k \leq 194$, be the irreducible characters of $\mathbb{M}$, which will often be used to denote the irreducible representations also. For the first few $\mathbb{V}_{\beth}$ 's, we have the decompositions :

$$
\begin{gathered}
\mathbb{V}_{\nvdash}=\chi_{\nVdash}, \\
\mathbb{V}_{\nvdash}=\chi_{\nVdash}+\chi_{\nvdash}, \\
\mathbb{V}_{\nVdash}=\chi_{\nVdash}+\chi_{\nvdash}+\chi_{\nVdash} .
\end{gathered}
$$

In general, write

$$
\mathbb{V}_{\bar{\sim}}=\sum_{\urcorner=\nVdash}^{\nVdash \nrightarrow \Phi} \bar{\sim} \backslash \chi_{\urcorner}
$$

where $c_{h k}$ is the multiplicity of $\chi_{k}$ in $\mathbb{V}_{\sim}$. The table of $c_{h k}$ for $0 \leq h \leq 51,1 \leq k \leq$ 194 can be found in McKay-Strauss [6].

We also list some of the multiplicities $c_{h 1}$ of the trivial character $\chi_{1}$ in $V_{h}$.

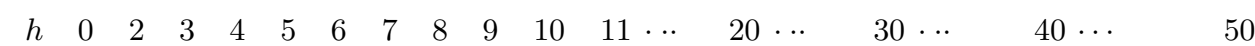

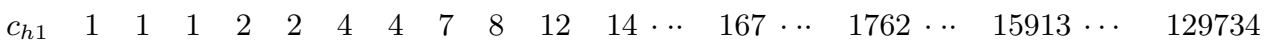

Let us consider, for each irreducible character $\chi_{k}$, the generating function :

$$
t_{\chi_{k}}(x)=x^{-1} \sum_{h=0}^{\infty} c_{h k} x^{h} .
$$

1991 Mathematics Subject Classification. 20D08; Secondary 11F03.

Key words and phrases. monster, monster module, modular functions, invariance groups. 
The multiplicity $c_{h k}$ can be computed as follows :

$$
c_{h k}=\frac{1}{|\mathbb{M}|} \sum_{g \in \mathbb{M}} \operatorname{Tr}\left(g \mid \mathbb{V}_{\bar{\sim}}\right) \chi_{\urcorner}(\check{\partial}) .
$$

Therefore the generating function of $c_{h k}$ is

$$
t_{\chi_{k}}(x)=x^{-1} \sum_{h=1}^{\infty} \sum_{g \in \mathbb{M}} \frac{1}{|\mathbb{M}|} \operatorname{Tr}\left(g \mid \mathbb{V}_{\bar{\sim}}\right) \chi_{\urcorner}(ð) \curvearrowleft^{\bar{\sim}} .
$$

If we replace the indeterminate $x$ by $q=e^{2 \pi i z}, z \in \mathbb{H}=\{z \in \mathbb{C} \mid \operatorname{Im}(\digamma)>\nvdash\}$ then

$$
t_{\chi_{k}}(q)=\frac{1}{|\mathbb{M}|} \sum_{g \in \mathbb{M}} \chi_{k}(g) t_{g}(q)
$$

where

$$
t_{g}(q)=q^{-1} \sum_{h=0}^{\infty} \operatorname{Tr}\left(g \mid \mathbb{V}_{\bar{\sim}) ॥} \bar{\sim}\right.
$$

is the McKay-Thompson series for the element $g$ in $\mathbb{M}$. Thus $t_{\chi}(q)$ for the irreducible character $\chi$ is the weighted sum of the McKay-Thompson series for the element $g$ of M. Not all $t_{\chi}(q)$ 's are distinct and in fact there are exactly 172 distinct $t_{\chi}(q)$ 's, since

$$
t_{\chi}(q)=t_{\bar{\chi}}(q)
$$

where $\bar{\chi}$ is the complex conjugate of $\chi$ and there is no other equalities among $t_{\chi}(q)$ 's. One of the obvious questions one will raise here will be :

Problem. Determine the invariance group $\Gamma_{\chi}$ of $t_{\chi}(q)$.

Here $\Gamma_{\chi}$ is defined to be :

Definition. $\Gamma_{\chi}=\left\{A \in S L_{2}(\mathbb{R}) \mid \approx_{\chi}(\mathbb{A} \digamma)=\approx_{\chi}(\digamma)\right\}$.

Since $t_{\chi}(z)$ is a modular function, $\Gamma_{\chi}$ is a discrete subgroup of $S L_{2}(\mathbb{R})$. Let us here review some of the properties of the invariance group $\Gamma_{g}$ of the McKayThompson series $t_{g}(z)$ for the element $g \in \mathbb{M}$.

(0). For $G \subset G L_{2}^{+}(\mathbb{R}), \bar{G}$ is the image of $G$ in $P G L_{2}^{+}(\mathbb{R})$.

(1). $\Gamma_{0}(N)=\left\{A=\left(\begin{array}{ll}a & b \\ c & d\end{array}\right) \in S L_{2}(\mathbb{Z}) \mid \equiv \nvdash(\bmod N)\right\}$.

(2). For an exact divisor $e \| N$ (i.e. $e \mid N$ and $\operatorname{gcd}\left(e, \frac{N}{e}\right)=1$ ) let

$$
W_{e}=\left(\begin{array}{ll}
a e & b \\
c N & d e
\end{array}\right), \quad a, b, c, d \in \mathbb{Z}, \quad \partial^{\not \models}-\mathbb{N}=.
$$

Then $\bar{W}_{e}$ normalizes $\bar{\Gamma}_{0}(N)$ and $\bar{W}_{e}^{2} \in \bar{\Gamma}_{0}(N)$.

(3). Let $h$ be a divisor of $n$. Then $n \mid h+e, f, \cdots$ is defined to be

$$
\left(\begin{array}{cc}
\frac{1}{h} & 0 \\
0 & 1
\end{array}\right)\left\langle\Gamma_{0}\left(\frac{n}{h}\right), W_{e}, W_{f}, \cdots\right\rangle\left(\begin{array}{cc}
h & 0 \\
0 & 1
\end{array}\right) .
$$

(4). For each $g$ in $\mathbb{M}, \Gamma_{g}$, the invariance group of $t_{g}(z)$, is a normal subgroup of index $h_{g}$ in $n_{g} \mid h_{g}+e_{g}, f_{g}, \cdots$, the eigen group of $g$ [2]. Note that for each $A$ in $n_{g} \mid h_{g}+e_{g}, f_{g}, \cdots,\left(t_{g} \mid A\right)(z)=\sigma t_{g}(z)$ where $\sigma$ is an $h_{g}$-th root of unity. We will often use $n, h, e, f$, etc. instead of $n_{g}, h_{g} e_{g}, f_{g}$, etc. for simplicity.

(5). $\Gamma_{g}$ contains $\Gamma_{0}\left(n_{g} h_{g}\right)$. 
For each irreducible character of $\mathbb{M}$, we now define :

Definition. $N_{\chi}=\operatorname{lcm}\left\{n_{g} h_{g} \mid g \in \mathbb{M}, \chi(ð) \neq \nvdash\right\}$.

It is obvious that $t_{\chi}(z)$ is invariant under $\Gamma_{0}\left(N_{\chi}\right)$. Note that $N_{\chi}$ can be quite large $\left(N_{\chi_{1}}=2^{6} 3^{3} 5^{2} 7 \cdot 11 \cdot 13 \cdot 17 \cdot 19 \cdot 23 \cdot 29 \cdot 31 \cdot 41 \cdot 47 \cdot 59 \cdot 71\right)$ or relatively small $\left(N_{\chi_{166}}=2^{6} 3^{2} 7=4032\right)$.

The purpose of this paper is to show

Theorem. $\Gamma_{\chi}=\Gamma_{0}\left(N_{\chi}\right)$.

\section{Poles of $t_{\chi}(z)$}

For each cusp $c$ in $\mathbb{Q} \cup\{\infty\}$, we define $\Phi_{c}$ to be the set $\Phi_{c}=\{g \in \mathbb{M} \mid$ is equivalent to $\infty$ in $\Gamma_{g}$ \} and decompose $t_{\chi}(z)$ into :

$$
\frac{1}{|\mathbb{M}|} \sum_{g \in \Phi_{c}} \chi(g) t_{g}(z)+\frac{1}{|\mathbb{M}|} \sum_{g \notin \Phi_{c}} \chi(g) t_{g}(z) .
$$

Since the McKay-Thompson series $t_{g}(z)$ is a generator of the function field of the compact Riemann surface $\Gamma_{g} \backslash \mathbb{H}^{*}\left(\mathbb{H}^{*}=\mathbb{H} \cup\{\infty\}\right)$ of genus 0 and has a unique pole at $\infty$ (and at all cusps $c \in \mathbb{Q}$ equivalent to $\infty$ in $\Gamma_{g}$ ). Obviously, $\frac{1}{|\mathbb{M}|} \sum_{g \notin \Phi_{c}} \chi(g) t_{g}(z)$ is holomorphic at $c$. Hence, whether $c$ is a pole of $t_{\chi}(z)$ or not is determined by the singular part of

$$
\frac{1}{|\mathbb{M}|} \sum_{g \in \Phi_{c}} \chi(g) t_{g}(z)
$$

at $c$. For example, if $c=\infty$, then $\infty$ is a pole of $t_{\chi}(z)$ if and only if $\chi$ is the trivial character since the singular part of $t_{\chi}(z)$ at $\infty$ is given by $\frac{1}{|\mathbb{M}|} \sum_{g \in \mathbb{M}} \chi(g) \frac{1}{q}$ and

$$
\sum_{g \in \mathbb{M}} \chi_{i}(g)= \begin{cases}|\mathbb{M}| & \text { if } i=1 \\ 0 & \text { if } i \neq 1\end{cases}
$$

Suppose $c \in \mathbb{Q}$. For each $g$ in $\Phi_{c}$, let $A \in S L_{2}(\mathbb{Z})$ be chosen so that $A \infty=c$. Then $t_{g}(A z)=\left(t_{g} \mid A\right)(z)$ has an expansion in $q=e^{2 \pi i z}$ of the form

$$
a q^{-\frac{1}{\mu}}+\cdots
$$

where $\mu=\left[\left\langle\left(\begin{array}{ll}1 & 1 \\ 0 & 1\end{array}\right)\right\rangle: A^{-1} \Gamma_{0}\left(n_{g} h_{g}\right)_{c} A\right]$, where the subscript $c$ denotes the stabilizer. We contend that the contribution of the $t_{g}(z)$ to the singular part of $t_{\chi}(z)$ is

$$
a q^{-\frac{1}{\mu}}
$$

Indeed, by our assumption the cusp $c$ is equivalent to $\infty$ in $\Gamma_{g}$ and so there is $B \in \Gamma_{g}$ such that $B \infty=c$ and

$$
\left(t_{g} \mid B\right)(q)=t_{g}(q)=q^{-1}+\sum_{i \geq 0} a_{i} q^{i} .
$$

The only difference between $\left(t_{g} \mid A\right)(z)$ and $\left(t_{g} \mid B\right)(z)$ lies in the power of $q$ and $a$, hence the contention.

In order to determine whether $c$ is pole of $t_{\chi}(z)$ or not, one has to :

(1). Determine whether $c$ is equivalent to $\infty$ in $\Gamma_{g}$ or not.

(2). Determine the singular part of $\frac{1}{|\mathbb{M}|} \sum_{g \in \Phi_{c}} \chi(g) t_{g}(z)$ at $c$. 
We will investigate those questions in the next section.

\section{EQUIVALENCE OF CUSPS}

In this section, we will study the equivalence of cusps in $\Gamma_{g}, g \in \mathbb{M}$.

Lemma 1. For each exact divisor e of $N$ and for each $c$ such that $\operatorname{gcd}(c, e)=1$, $\Gamma_{0}(N)$ admits an Atkin-Lehner involution of the form $W_{e}=\left(\begin{array}{ll}a e & b \\ c N & d e\end{array}\right)$. Moreover, one can choose either $a=1$ or $d=1$ if desired.

Proof. For each $c$ such that $c$ and $e$ are relatively prime, we have $\operatorname{gcd}\left(\frac{c N}{e}, e\right)=1$. Hence, there exists $b$ and $y$ such that $y e-\frac{b c N}{e}=1$, or $y e^{2}-b c N=e$. The lemma follows by writing $y$ into $a d$ for suitable $a$ and $d$.

Lemma 2. Let $\operatorname{gcd}(a, b)=1$ and $M$ be nonzero integers. Then there exists a pair of integers $x$ and $y$ satisfying $\operatorname{gcd}(x M, y)=1$ and $a x+b y=1$.

Proof. This is a well known fact of the elementary number theory. Let $x^{\prime}$ and $y^{\prime}$ be a pair of integral solutions of the equation $a x+b y=1$ and let $M=M_{a} M_{y^{\prime}} M^{\prime}$ be the decomposition of $M$ into a product of coprime factors such that $M_{a}$ and $a, M_{y^{\prime}}$ and $y^{\prime}$, have the same prime factors. It is clear that $y=y^{\prime}+a M^{\prime}$ and $x=x^{\prime}-b M^{\prime}$ is also a pair of solutions to the equation. Note that $\operatorname{gcd}(x, y)=1$ since it is a solution of $a x+b y=1$. Furthermore, one has $\operatorname{gcd}(y, M)=\operatorname{gcd}\left(y^{\prime}+a M^{\prime}, M_{a} M_{y^{\prime}} M^{\prime}\right)$ $=\operatorname{gcd}\left(y^{\prime}+a M^{\prime}, M^{\prime}\right)=1$. Therefore $x$ and $y$ is pair of integral solutions of the equation such that $\operatorname{gcd}(x M, y)=1$.

Lemma 3. Let $\frac{x}{y}, \operatorname{gcd}(x, y)=1$, be a rational number. Then $\frac{x}{y}$ is equivalent to some $\frac{x^{\prime}}{y^{\prime}}, \operatorname{gcd}\left(x^{\prime}, y^{\prime}\right)=1$ in $\Gamma_{0}(N)$, where $y^{\prime}=\operatorname{gcd}(N, y)$. Furthermore, if $\frac{x}{y}$ is equivalent to $\frac{x^{\prime \prime}}{y^{\prime \prime}}$ with $y^{\prime \prime} \mid N, y^{\prime \prime}>0$, and $\operatorname{gcd}\left(x^{\prime \prime}, y^{\prime \prime}\right)=1$, then $y^{\prime \prime}=y^{\prime}$.

Proof. Consider the equality

$$
\left(\begin{array}{ll}
a & b \\
c N & d
\end{array}\right) \frac{x}{y}=\frac{a x+b y}{c N x+d y}=\frac{a x+d y}{y^{\prime}\left(c x \frac{N}{y^{\prime}}+d \frac{y}{y^{\prime}}\right)}
$$

Note that $\operatorname{gcd}\left(x \frac{N}{y^{\prime}}, \frac{y}{y^{\prime}}\right)=1$, hence the equation

$$
c \frac{x N}{y^{\prime}}+d \frac{y}{y^{\prime}}=1
$$

is solvable for $c, d$ in $\mathbb{Z}$. Applying Lemma 2, we may assume that $c$ and $d$ are integral solutions of the above equation such that $\operatorname{gcd}(c N, d)=1$. Let $a$ and $b$ be chosen such that $a d-c b N=1$. Summerizing, we now conclude that $\frac{x}{y}$ is equivalent to $\frac{a x+b y}{y^{\prime}}$ by $\left(\begin{array}{cc}a & b \\ c N & d\end{array}\right) \in \Gamma_{0}(N)$. Since $\operatorname{gcd}\left(a x+b y, y^{\prime}\right)=\operatorname{gcd}\left(a x, y^{\prime}\right)=\operatorname{gcd}\left(a, y^{\prime}\right)=1$ , first part of the lemma is proved. As for the second part, suppose

$$
\left(\begin{array}{ll}
a & b \\
c N & d
\end{array}\right) \frac{x}{y}=\frac{a x+b y}{c N x+d y}=\frac{a x+d y}{y^{\prime}\left(c x \frac{N}{y^{\prime}}+d \frac{y}{y^{\prime}}\right)}=\frac{x^{\prime \prime}}{y^{\prime \prime}} .
$$

We first note $y^{\prime} \mid y^{\prime \prime}$, since $\operatorname{gcd}\left(a x+b y, y^{\prime}\right)=1$. To show $y^{\prime \prime} \mid y^{\prime}$, suppose that $y^{\prime \prime}$ possesses a prime power $q^{t}$ such that $y^{\prime}$ is not a multiple of $q^{t}$, then $q^{t} \mid y^{\prime}\left(c x \frac{N}{y^{\prime}}+d \frac{y}{y^{\prime}}\right)$ 
implies $q \mid\left(c x \frac{N}{y^{\prime}}+d \frac{y}{y^{\prime}}\right)$. Since $y^{\prime \prime} \mid N, q$ is a divisor of $\frac{N}{y^{\prime}}$, hence $q \mid d$. This implies that $\operatorname{gcd}(c N, d) \neq 1$, against our choice of $c, d$. Thus, $y^{\prime \prime} \mid y^{\prime}$ and the second part of the lemma is proved.

In Lemma 4 and Lemma $5, G=n \mid h+e, f, \cdots$ is the eigen group of the invariance group $\Gamma_{g}$.

Lemma 4. Let $g \in \mathbb{M}$ and let $\Gamma_{g} \leq G=n \mid h+e, f, \cdots$ be the invariance group of $t_{g}(z)$. Then $G \infty=\Gamma_{g} \infty$.

Proof. Since $q=\exp (2 \pi i z)$ is a local parameter of $t_{g}(z)$, the stabilizer $\left(\Gamma_{g}\right)_{\infty}$ of $\infty$ is generated by $\left(\begin{array}{ll}1 & 1 \\ 0 & 1\end{array}\right)$. As for $G, G_{\infty}$ is generated by $\left(\begin{array}{cc}1 & \frac{1}{h} \\ 0 & 1\end{array}\right)$. Hence

$$
\left[G: \Gamma_{g}\right]=h=\left[G_{\infty}:\left(\Gamma_{g}\right)_{\infty}\right]
$$

Consequently, $G \infty=\Gamma_{g} \infty$.

Lemma 5. Let $g \in \mathbb{M}$ and let $\Gamma_{g} \leq n \mid h+e, f, \cdots$ be the invariance group of $t_{g}(z)$. Then $\frac{x}{y}, \operatorname{gcd}(x, y)=1$, is equivalent to $\infty$ in $\Gamma_{g}$ if and only if

$$
\operatorname{gcd}\left(\frac{n}{h}, \frac{y}{\operatorname{gcd}(y, h)}\right) \in\left\{\frac{n}{h}, \frac{n}{h e}, \frac{n}{h f}, \cdots\right\}
$$

Proof. To simplify our notation, let $\mathrm{N}=\frac{n}{h}$. Choose the Atkin-Lehner involution $W_{e}$ as described in Lemma 1 with $\operatorname{gcd}(c, N)=1$. One has $W_{e} \infty=\frac{a}{c \frac{N}{e}}$, and

$$
\operatorname{gcd}\left(a, c \frac{N}{e}\right)=1 \text {. }
$$

By Lemma 3, there exists $\gamma_{e} \in \Gamma_{0}(N)$ such that $\gamma_{e} W_{e} \infty=\frac{e^{\prime}}{\frac{N}{e}}$ where $\operatorname{gcd}\left(e^{\prime}, \frac{N}{e}\right)=1$, since $\operatorname{gcd}\left(c \frac{N}{e}, N\right)=\frac{N}{e}$. Note that $e$ is an exact divisor of $N$, hence among the representitives of inequivalent cusps of $\Gamma_{0}(N)$, there is exactly one and only one cusp $z$ with denominator $\frac{N}{e}$ (see Harada [4]). Without loss of generality, we may assume that $z=\frac{1}{\frac{N}{e}}$. Therefore, we may assume that $\gamma_{e}$ is chosen so that $\gamma_{e} W_{e} \infty=\frac{1}{\frac{N}{e}}$. Hence the $G$-orbit of $\infty$ can be decomposed into,

$$
\left(\begin{array}{cc}
\frac{1}{h} & 0 \\
0 & 1
\end{array}\right) \Gamma_{0}(N) \frac{1}{N} \cup\left(\begin{array}{cc}
\frac{1}{h} & 0 \\
0 & 1
\end{array}\right) \Gamma_{0}(N) \frac{1}{\frac{N}{e}} \cup\left(\begin{array}{cc}
\frac{1}{h} & 0 \\
0 & 1
\end{array}\right) \Gamma_{0}(N) \frac{1}{\frac{N}{f}} \cup \cdots .
$$

Hence $\frac{x}{y}$ is equivalent to $\infty$ in $G$ if and only if

$$
\left(\begin{array}{cc}
h & 0 \\
0 & 1
\end{array}\right) \frac{x}{y}=\frac{\frac{h x}{\operatorname{gcd}(y, h)}}{\frac{y}{\operatorname{gcd}(y, h)}} \in \Gamma_{0}(N) \frac{1}{N} \cup \Gamma_{0}(N) \frac{1}{\frac{N}{e}} \cup \Gamma_{0}(N) \frac{1}{\frac{N}{f}} \cup \cdots,
$$

which is equivalent to, by Lemma 3 ,

$$
\operatorname{gcd}\left(\frac{y}{\operatorname{gcd}(y, h)}, N\right) \in\left\{N, \frac{N}{e}, \frac{N}{f}, \cdots\right\} .
$$

$G \infty=\Gamma_{g} \infty$ as shown in Lemma 4 and so $\frac{x}{y}$ is equivalent to $\infty$ in $\Gamma_{g}$ if and only if

$$
\operatorname{gcd}\left(\frac{y}{\operatorname{gcd}(y, h)}, \frac{n}{h}\right) \in\left\{\frac{n}{h}, \frac{n}{h e}, \frac{n}{h f}, \cdots\right\} \text {. }
$$


Corollary 6. $0=\frac{0}{1}$ is equivalent to $\infty$ in $\Gamma_{g}$ if and only if $n=h$ or $G=$ $n \mid h+e, f, \cdots$ admits the Atkin-Lehner involution $W_{\frac{n}{h}}$.

Proof. Since $\operatorname{gcd}\left(1, \frac{n}{h}\right)=1, G$ must admits an Atkin-Lehner involution $W_{e}$ such that $\frac{n}{h e}=1$, hence $e=\frac{n}{h}$.

Let $\chi$ be an irreducible character of the monster $\mathbb{M}$. In order to determine the singular part of

$$
\frac{1}{|\mathbb{M}|} \sum_{g \in \Phi_{c}} \chi(g) t_{g}(z)
$$

at the cusp $c=\frac{x}{y}$, where $\operatorname{gcd}(x, y)=1$ and $y \mid N_{\chi}$, it is necessary to find a matrix $P_{c}$ in $S L_{2}(\mathbb{R})$ such that $P_{c} \infty=c$ and determine the $q$-expansion of

$$
\frac{1}{|\mathbb{M}|} \sum_{g \in \Phi_{c}} \chi(g)\left(t_{g} \mid P_{c}\right)(z)
$$

which will be called the $q$-expansion of $t_{\chi}(z)$ at $c$. Such a matrix $P_{c}$ is easy to find and choice is not unique. To ease the computation of the $q$-expansion of

$$
\frac{1}{|\mathbb{M}|} \sum_{g \in \Phi_{c}} \chi(g)\left(t_{g} \mid P_{c}\right)(z)
$$

it is necessary to find a good $P_{c}$ so that the transformation formula of $t_{g} \mid P_{c}$ can be obtained for every $g$ in $\Phi_{c}$ simultaneously. What we shall do is as follows. Namely, we will find a matrix $P_{c}$ so that one can associate with $P_{c}$ an upper triangular matrix $U_{c, g}$ such that

$$
P_{c} U_{c, g}^{-1} \in n_{g} \mid h_{g}+e_{g}, f_{g}, \cdots
$$

Since $n_{g} \mid h_{g}+e_{g}, f_{g}, \cdots$ is the eigen group of $\Gamma_{g}$, elements in $n_{g} \mid h_{g}+e_{g}, f_{g}, \cdots$ map $t_{g}$ to $\sigma_{g} t_{g}$ where $\sigma_{g}$ is an $h_{g}$-th root of unity which depends on $g$ and on some other quantities. Therefore

$$
t_{g}\left|P_{c}=\sigma_{g} t_{g}\right| U_{c, g}
$$

A good transformation formula for $t_{g} \mid P_{c}$ is obtained since $U_{c, g}$ is upper trianglar.

Let $y_{0}$ be the exact divisor of $N_{\chi}$ such that $y$ and $y_{0}$ share the same prime divisors. Then $\operatorname{gcd}\left(y, x \frac{N_{\chi}}{y_{0}}\right)=1$ and so there is a matrix $P_{c} \in S L_{2}(\mathbb{Z})$ of the form

$$
P_{c}=\left(\begin{array}{ll}
x & w \\
y & \frac{z N_{\chi}}{y_{0}}
\end{array}\right) \text {. }
$$

Lemma 4 implies that $\frac{x}{y}$ is equivalent to $\infty$ in $\Gamma_{g}$ if and only if $\frac{x}{y}$ is equivalent to $\infty$ in the eigen group $n_{g} \mid h_{g}+e_{g}, f_{g}, \cdots$ of $g$ and so by Lemma $5, \frac{x}{y}$ is equivalent to $\infty$ in $\Gamma_{g}$ if and only if $\operatorname{gcd}\left(\frac{n_{g}}{h_{g}}, \frac{y}{\operatorname{gcd}\left(y, h_{g}\right)}\right) \in\left\{\frac{n}{h}, \frac{n}{h e}, \frac{n}{h f}, \cdots\right\}$. More precisely, $\frac{x}{y}$ is equivalent to $\infty$ by an element in $n_{g} \mid h_{g}$ if

$$
\operatorname{gcd}\left(\frac{n_{g}}{h_{g}}, \frac{y}{\operatorname{gcd}\left(y, h_{g}\right)}\right)=\frac{n_{g}}{h_{g}}
$$

and is equivalent to $\infty$ by an Atkin-Lehner involution $W_{e_{g}}$ of $n_{g} \mid h_{g}+e_{g}, f_{g}, \cdots$ if

$$
\frac{n_{g}}{h_{g} e_{g}}=\operatorname{gcd}\left(\frac{n_{g}}{h_{g}}, \frac{y}{\operatorname{gcd}\left(y, h_{g}\right)}\right) \in\left\{\frac{n}{h e}, \frac{n}{h f}, \cdots\right\} \text {. }
$$


Lemma 7. Suppose that $\operatorname{gcd}\left(\frac{n_{g}}{h_{g}}, \frac{y}{\operatorname{gcd}\left(y, h_{g}\right)}\right)=\frac{n_{g}}{h_{g}}$. Let $u_{g}$ be chosen so that $\frac{y u_{g}}{h_{g}}+$ $\frac{z N_{\chi} g c d\left(h_{g}, y\right)}{y_{0} h_{g}}$ is an integer. Then

$$
P_{c} U_{c, g}^{-1}=P_{c}\left(\begin{array}{ll}
\frac{h_{g}}{g c d\left(h_{g}, y\right)} & \frac{u_{g}}{h_{g}} \\
0 & \frac{g c d\left(h_{g}, y\right)}{h_{g}}
\end{array}\right) \in n_{g} \mid h_{g}
$$

where

$$
U_{c, g}=\left(\begin{array}{ll}
\frac{g c d\left(h_{g}, y\right)}{h_{g}} & -\frac{u_{g}}{h_{g}} \\
0 & \frac{h_{g}}{g c d\left(h_{g}, y\right)}
\end{array}\right)
$$

Proof. To show the existence of $u_{g}$, simply solve the equation

$$
\frac{y}{\operatorname{gcd}\left(h_{g}, y\right)} u_{g}+\frac{N_{\chi}}{y_{0}} z \equiv 0 \quad\left(\bmod \frac{h_{g}}{\operatorname{gcd}\left(h_{g}, y\right)}\right)
$$

Then $y u_{g}+z \frac{N_{\chi}}{y_{0}} \operatorname{gcd}\left(h_{g}, y\right) \equiv 0\left(\bmod h_{g}\right)$ and hence $\frac{y u_{g}}{h_{g}}+\frac{z N_{\chi} \operatorname{gcd}\left(h_{g}, y\right)}{y_{0} h_{g}}$ is an integer.

The matrix

$$
P_{c}\left(\begin{array}{ll}
\frac{h_{g}}{\operatorname{gcd}\left(h_{g}, y\right)} & \frac{u_{g}}{h_{g}} \\
0 & \frac{\operatorname{gcd}\left(h_{g}, y\right)}{h_{g}}
\end{array}\right)=\left(\begin{array}{ll}
\frac{x h_{g}}{\operatorname{gcd}\left(h_{g}, y\right)} & \frac{x u_{g}+w \operatorname{gcd}\left(h_{g}, y\right)}{h_{g}} \\
\frac{y h_{g}}{\operatorname{gcd}\left(h_{g}, y\right)} & \frac{y u_{g}}{h_{g}}+\frac{z N_{\chi} \operatorname{gcd}\left(h_{g}, y\right)}{y_{0} h_{g}}
\end{array}\right)
$$

has the property

(1). $\frac{y u_{g}}{h_{g}}+\frac{z N_{\chi} \operatorname{gcd}\left(h_{g}, y\right)}{y_{0} h_{g}}$ is an integer by our choice of $u_{g}$, and,

(2). $\frac{y h_{g}}{\operatorname{gcd}\left(h_{g}, y\right)}$ is a multiple of $n_{g}$ since $\operatorname{gcd}\left(\frac{n_{g}}{h_{g}}, \frac{y}{\operatorname{gcd}\left(y, h_{g}\right)}\right)=\frac{n_{g}}{h_{g}}$.

Therefore

$$
P_{c} U_{c, g}^{-1}=P_{c}\left(\begin{array}{ll}
\frac{h_{g}}{\operatorname{gcd}\left(h_{g}, y\right)} & \frac{u_{g}}{h_{g}} \\
0 & \frac{\operatorname{gcd}\left(h_{g}, y\right)}{h_{g}}
\end{array}\right) \in n_{g} \mid h_{g}
$$

Corollary 8. Suppose that $\operatorname{gcd}\left(\frac{n_{g}}{h_{g}}, \frac{y}{\operatorname{gcd}\left(y, h_{g}\right)}\right)=\frac{n_{g}}{h_{g}}$. Then

$$
t_{g}\left|P_{c}=\sigma_{g} t_{g}\right| U_{c, g}=\sigma_{g} t_{g}\left(U_{c, g} z\right)=\sigma_{g} t_{g}\left(\frac{g c d\left(h_{g}, y\right)^{2}}{h_{g}{ }^{2}} z-\frac{u_{g}}{h_{g}^{2}} g c d\left(h_{g}, y\right)\right)
$$

where $\sigma_{g}$ is an $h_{g}$-th root of unity.

Proof. Since $P_{c}\left(\begin{array}{ll}\frac{h_{g}}{\operatorname{gcd}\left(h_{g}, y\right)} & \frac{u_{g}}{h_{g}} \\ 0 & \frac{\operatorname{gcd}\left(h_{g}, y\right)}{h_{g}}\end{array}\right) \in n_{g} \mid h_{g}$ and $n_{g} \mid h_{g}$ is the eigen group of $t_{g}(z)$

$$
t_{g} \mid P_{c}\left(\begin{array}{ll}
\frac{h_{g}}{\operatorname{gcd}\left(h_{g}, y\right)} & \frac{u_{g}}{h_{g}} \\
0 & \frac{\operatorname{gcd}\left(h_{g}, y\right)}{h_{g}}
\end{array}\right)=\sigma_{g} t_{g}(z) .
$$

This completes the proof of the corollary.

We now consider the case that $c$ is equivalent to $\infty$ in the eigen group of $\Gamma_{g}$ by an Atkin-Lehner involution $W_{e_{g}}$. 
Lemma 9. Suppose that $c=\frac{x}{y}$ is equivalent to $\infty$ in the eigen group of $\Gamma_{g}$ by an Atkin-Lehner involution $W_{e_{g}}$. Let an integer $u_{g}$ be chosen such that $e_{g}$ is a divisor of an integer $\frac{u_{g} y}{h_{g}}+\frac{z N_{\chi} g c d\left(h_{g}, y\right)}{h_{g} y_{0}}$ where

$$
e_{g}=\frac{\frac{n_{g}}{h_{g}}}{\operatorname{gcd}\left(\frac{n_{g}}{h_{g}}, \frac{y}{g c d\left(y, h_{g}\right)}\right)} .
$$

Then

$$
P_{c} U_{c, g}^{-1}=P_{c}\left(\begin{array}{ll}
\frac{e_{g} h_{g}}{g c d\left(h_{g}, y\right)} & \frac{u_{g}}{h_{g}} \\
0 & \frac{g c d\left(h_{g}, y\right)}{h_{g}}
\end{array}\right)=W_{e_{g}} \in n_{g} \mid h_{g}+e_{g}, f_{g}, \cdots
$$

Furthermore,

$$
t_{g} \mid P_{c}=\sigma_{g} t_{g}\left(U_{c, g} z\right)=\sigma_{g} t_{g}\left(\frac{g c d\left(h_{g}, y\right)^{2}}{e_{g} h_{g}{ }^{2}} z-\frac{u_{g}}{e_{g} h_{g}^{2}} \operatorname{gcd}\left(h_{g}, y\right)\right)
$$

where $\sigma_{g}$ is an $h_{g}$-th root of unity.

Proof. Let us first show that such an $u_{g}$ exists. We will need $u_{g}$ such that

$$
y u_{g}+z \frac{N_{\chi}}{y_{0}} \operatorname{gcd}\left(h_{g}, y\right) \equiv 0 \quad\left(\bmod e_{g} h_{g}\right) .
$$

This follows from

$$
\frac{y}{\operatorname{gcd}\left(h_{g}, y\right)} u_{g}+z \frac{N_{\chi}}{y_{0}} \equiv 0 \quad\left(\bmod \frac{h_{g}}{\operatorname{gcd}\left(h_{g}, y\right)} e_{g}\right) .
$$

Since $\operatorname{gcd}\left(\frac{n_{g}}{h_{g}}, \frac{y}{\operatorname{gcd}\left(h_{g}, y\right)}\right)=\frac{n_{g}}{h_{g} e_{g}}$ and $e_{g}$ is an exact divisor of $\frac{n_{g}}{h_{g}}$, we see that

$$
\operatorname{gcd}\left(\frac{y}{\operatorname{gcd}\left(h_{g}, y\right)}, e_{g}\right)=1 .
$$

Therefore $\frac{y}{\operatorname{gcd}\left(h_{g}, y\right)}$ is invertible modulo $\frac{h_{g}}{\operatorname{gcd}\left(h_{g}, y\right)} e_{g}$, hence $u_{g}$ exists as required.

The matrix

$$
P_{c}\left(\begin{array}{ll}
\frac{e_{g} h_{g}}{\operatorname{gcd}\left(h_{g}, y\right)} & \frac{u_{g}}{h_{g}} \\
0 & \frac{\operatorname{gcd}\left(h_{g}, y\right)}{h_{g}}
\end{array}\right)=\left(\begin{array}{ll}
\frac{x e_{g} h_{g}}{\operatorname{gcd}\left(h_{g}, y\right)} & \frac{x u_{g}+w \operatorname{gcd}\left(h_{g}, y\right)}{h_{g}} \\
\frac{y e_{g} h_{g}}{\operatorname{gcd}\left(h_{g}, y\right)} & \frac{y u_{g}}{h_{g}}+\frac{z N_{\chi} \operatorname{gcd}\left(h_{g}, y\right)}{y_{0} h_{g}}
\end{array}\right)
$$

has the property

(1). $\frac{u_{g} y}{h_{g}}+\frac{z N_{\chi} \operatorname{gcd}\left(h_{g}, y\right)}{h_{g} y_{0}}$ is a multiple of $e_{g}$ by choice of $u_{g}$, and,

(2). $\frac{y e_{g} h_{g}}{\operatorname{gcd}\left(h_{g}, y\right)}$ is a multiple of $n_{g}$, since $\operatorname{gcd}\left(\frac{n_{g}}{h_{g}}, \frac{y}{\operatorname{gcd}\left(y, h_{g}\right)}\right)=\frac{n_{g}}{h_{g} e_{g}}$.

Therefore

$$
P_{c}\left(\begin{array}{ll}
\frac{e_{g} h_{g}}{\operatorname{gcd}\left(h_{g}, y\right)} & \frac{u_{g}}{h_{g}} \\
0 & \frac{\operatorname{gcd}\left(h_{g}, y\right)}{h_{g}}
\end{array}\right)=W_{e_{g}} \in n_{g} \mid h_{g}+e_{g}, f_{g}, \cdots .
$$

Since $c$ is equivalent to $\infty$ in the eigen group of $\Gamma_{g}$ by $W_{e_{g}}$, the transformation formula follows easily.

Remark. It is easy to see that Lemma 7 and Corollary 8 are included in Lemma 9 if $e_{g}=1$, in which case every element of $n_{g} \mid h_{g}$ is called an Atkin-Lehner involution for $e_{g}=1$. This abuse of words will be used occasionally for the balance of the paper. 
The singular part of $\frac{1}{|\mathbb{M}|} \sum_{g \in \Phi_{c}} \chi(g)\left(t_{g} \mid P_{c}\right)(z)$ at $z=\infty i$ is now determined by

$$
\operatorname{sing}_{P_{c}} t_{\chi}=\frac{1}{|\mathbb{M}|} \sum_{g \in \Phi_{c}} \chi(g) \frac{\sigma_{g}}{e^{2 \pi i U_{c, g} z}} \text {. }
$$

We give a few examples in the calculation of $\operatorname{sing}_{P_{c}} t_{\chi}$ 's. Note that the first example will be used later in the determination of the invariance groups.

Example 1. Suppose $c=\frac{0}{1}$. Then $\operatorname{sing}_{P_{0}} t_{\chi}=\frac{1}{|\mathbb{M}|} \sum_{g \in \Phi_{c}} \chi(g) \frac{\sigma_{g}}{q^{\bar{n} h_{g}}}$.

Proof. In this case $y=1$. We may choose $P_{0}=\left(\begin{array}{ll}x & w \\ y & \frac{x N_{\chi}}{y_{0}}\end{array}\right)=\left(\begin{array}{rr}0 & -1 \\ 1 & 0\end{array}\right)$ and $u_{g}=0$. If the condition of Corollary 8 holds, then $n_{g}=h_{g}$ and

$$
t_{g} \mid P_{0}=\sigma_{g} t_{g}\left(\frac{z}{h_{g}^{2}}\right)=\sigma_{g} t_{g}\left(\frac{z}{n_{g} h_{g}}\right) .
$$

The only $g \in \Phi_{0} \subseteq \mathbb{M}$ satisfying $n_{g}=h_{g}$ are $1 A$ and $3 C$. We have

$$
t_{1 A} \mid P_{0}=t_{1 A} \quad \text { and } \quad t_{3 C} \mid P_{0}=\sigma_{3 C} t_{3 C}\left(\frac{1}{9} z\right) .
$$

On the other hand, if the condition of Lemma 9 holds, then $n_{g}=e_{g} h_{g}$ and

$$
t_{g} \mid P_{0}=\sigma_{g} t_{g}\left(\frac{z}{e_{g} h_{g}^{2}}\right)=\sigma_{g} t_{g}\left(\frac{z}{n_{g} h_{g}}\right) .
$$

Note that for all the remaining $g \in \Phi_{0} \backslash\{1 A, 3 C\} \subseteq \mathbb{M}, 0$ is equivalent to $\infty$ in $\Gamma_{g}$ by the Atkin-Lehner involution $W_{\frac{n_{g}}{h_{g}}}$ and hence the condition of Lemma 9 holds.

Remark. Example 1 shows that 0 is a pole of the McKay-Thompson series $t_{\chi}(z)$ for every $\chi$, since $n_{g} h_{g} \neq 1$ for $g \neq 1$ and so the coefficient of $q^{-1}$ is nonzero.

Example 2. Let $\chi$ be the trivial character and let $c=\frac{1}{3}$. Then $P_{\frac{1}{3}}=$ $\left(\begin{array}{cc}1 & {\left[\frac{N_{0}}{81}\right]} \\ 3 & \frac{N_{0}}{27}\end{array}\right)\left([x]\right.$ is the integral part of $x$ and $\left.\frac{N_{0}}{27} \equiv 1(\bmod 3)\right)$ and $t_{84 A} \mid P_{\frac{1}{3}}$ $=\sigma_{84 A} t_{84 A}\left(\frac{1}{56} z+\frac{1}{2}\right)$.

Proof. We know $\Gamma_{84 A}<84 \mid 2+$. Since $\operatorname{gcd}\left(\frac{84}{2}, \frac{3}{\operatorname{gcd}(3,2)}\right)=3=\frac{84}{2 e}$, we see that $e$ is 14 , and can choose $u_{g}=-28$.

$$
P_{\frac{1}{3}}\left(\begin{array}{rr}
28 & -\frac{28}{2} \\
0 & \frac{1}{2}
\end{array}\right)=W_{14} \in 84 \mid 2+.
$$

The rest follows easily.

Remark. (1). The invariance group $\Gamma_{g}$ of the harmonics $n \mid h+e, f, \cdots$ are not fully determined. (For each $g$, one can write down a set of generators of the invariance group $\Gamma_{g}$ easily. But determining whether or not a given element in $S L_{2}(\mathbb{R})$ is a word of those generators is nontrivial.) Hence we have to settle for $\sigma_{g}$ being an $h_{g}$-th root of unity.

(2). $\sigma_{g}=1$ if $h_{g}=1$.

(3). Let $p \in\{11,17,19,23,29,31,41,47,59,71\}$. Applying Lemma 5, one can prove $\Phi_{0}=\Phi_{\frac{x}{p}}$ and $\Phi_{\frac{1}{32}}=\Phi_{\frac{1}{64}}$. 


\section{INVARIANCE GROUP}

Let $f$ be a modular function and let $K_{f}$ be a subgroup of the invariance group (in $\left.S L_{2}(\mathbb{R})\right) \Gamma_{f}$ of $f$ of finite index. We shall determine the invariance group as follows. Define

$C_{f}=$ the set of all cusps of $K_{f}$, and,

$C_{0}=\left\{c \in C_{f} \mid c\right.$ is a pole of $\left.f\right\}$.

Lemma 10. We have $\Gamma_{f} C_{f} \subseteq C_{f}$ and $\Gamma_{f} C_{0} \subseteq C_{0}$.

Proof. Since $\left[\Gamma_{f}: K_{f}\right]<\infty, C_{f}$ is also the set of all cusps of $\Gamma_{f}$. The second statement is obvious.

Lemma 11. Let $f$ be a modular function and let $\Gamma_{f}$ be its invariance group. Suppose that $K_{f} \leq \Gamma_{f}$. Let $\alpha=\frac{a_{1}}{c_{1}}\left(a_{1} \neq 0\right)$ and $\beta=\frac{a_{2}}{c_{2}}$ be two inequivalent cusps of $K_{f}$. Let

$$
M_{1}=\left(\begin{array}{ll}
a_{1} & b_{1} \\
c_{1} & d_{1}
\end{array}\right) \quad \text { and } \quad M_{2}=\left(\begin{array}{ll}
a_{2} & b_{2} \\
c_{2} & d_{2}
\end{array}\right) .
$$

Then $\frac{a_{1}}{c_{1}}$ and $\frac{a_{2}}{c_{2}}$ are equivalent with respect to $\Gamma_{f}$ if and only if the q-expansion of $\left.f\right|_{M_{1}}$ is derived from that of $\left.f\right|_{M_{2}}$ under the substitution $z \rightarrow a z+b$ for some numbers $a$ and $b$ (if $c_{i}=0$, then $a_{i}=1$ and $\left.\frac{a_{i}}{c_{i}}=\infty\right)$.

Proof. Let $A \in \Gamma_{f}$ be such that $A \alpha=\beta$. Define the matrix $B$ such that

$$
A=M_{2}\left(\begin{array}{rr}
1 & 0 \\
-\frac{c_{1}}{a_{1}} & 1
\end{array}\right) B
$$

Since $A \alpha=\beta$, it follows that $B \alpha=\alpha$. Hence

$$
B=\left(\begin{array}{ll}
1 & 0 \\
\frac{c_{1}}{a_{1}} & 1
\end{array}\right)\left(\begin{array}{ll}
m_{11} & m_{12} \\
0 & m_{22}
\end{array}\right)\left(\begin{array}{rr}
1 & 0 \\
-\frac{c_{1}}{a_{1}} & 1
\end{array}\right)
$$

for some $m_{11}, m_{12}$ and $m_{22}$. In particular,

$$
A=M_{2}\left(\begin{array}{ll}
m_{11} & m_{12} \\
0 & m_{22}
\end{array}\right)\left(\begin{array}{rr}
1 & 0 \\
-\frac{c_{1}}{a_{1}} & 1
\end{array}\right)
$$

and

$$
A \alpha=M_{2}\left(\begin{array}{ll}
m_{11} & m_{12} \\
0 & m_{22}
\end{array}\right) \infty .
$$

It follows that $\left.f\right|_{M_{1}}=\left.f\right|_{A M_{1}}=$

$f\left|M_{2}\left(\begin{array}{rr}1 & 0 \\ -\frac{c_{1}}{a_{1}} & 1\end{array}\right) B M_{1}=f\right| M_{2}\left(\begin{array}{ll}m_{11} & m_{12} \\ 0 & m_{22}\end{array}\right)\left(\begin{array}{ll}a_{1} & b_{1} \\ 0 & a^{\prime}\end{array}\right)=f \mid M_{2}\left(\begin{array}{ll}m_{11}^{\prime} & m_{12}^{\prime} \\ 0 & m_{22}^{\prime}\end{array}\right)$,

for some $b_{1}, a^{\prime}, m_{11}^{\prime}, m_{12}^{\prime}$ and $m_{22}^{\prime}$. Consequently, $\left.f\right|_{M_{1}}$ is derived from that of $\left.f\right|_{M_{2}}$ under the substitution $z \rightarrow a z+b$ where $a=\frac{m_{11}^{\prime}}{m_{22}^{\prime}}$ and $b=\frac{m_{12}^{\prime}}{m_{22}^{\prime}}$. Conversely, one sees easily that $\alpha$ and $\beta$ is equivalent to each other by

$$
M_{1}\left(\begin{array}{rr}
\frac{1}{a} & -\frac{b}{a} \\
0 & 1
\end{array}\right) M_{2}^{-1} \in \Gamma_{f} .
$$

The invariance group $\Gamma_{f}$ can now be determined as follows : 
(1). Determine $C_{f}$, the set of all cusps of $K_{f}$.

(2). Determine the subset $C_{0}$.

(3). Determine the $q$-expansion of $f$ at all $c_{i}$ in $C_{0}$ by suitable matrices $M_{i}$ such that $M_{i} \infty=c_{i}$.

(4). Apply Lemma 11 and determine the set $E_{0}=\left\{c \in C_{0} \mid\right.$ the $q$-expansion of $f$ at $c$ is derived from the $q$-expansion of $f$ at 0 under the substitution $z \rightarrow a z+b\}$ and the set $A_{0}=\left\{A_{c} \in \Gamma_{f}, A_{c} 0=c\right\}$. Note that it is sufficient to determine at most one matrix $A_{c}$ for each representative of inequivalent cusps.

(5). Determine $\left(\Gamma_{f}\right)_{0}=\langle B| B=\left(\begin{array}{ll}1 & 0 \\ m & 1\end{array}\right), m \in r \mathbb{Z}$, for some (fixed) $\left.\backslash \in \mathbb{Q}\right\rangle$. Note that this can be achieved by investigating the $q$-expansion of $f$ at 0 . Note also that $B$ is of the form given since $\Gamma_{f}$ is discrete.

Remark. (1). The McKay-Thompsom series $t_{\chi}(z)$ has a pole at 0 for every $\chi$ as stated in the remark right after Example 1.

(2). Since Lemma 11 applies only when one of the cusp is nonzero, one can not take $c$ to be 0 in (4) above.

(3). One can replace 0 by any cusp and apply our procedure to find the invariance groups.

Lemma 12. $\Gamma_{f}=\left\langle K_{f}, B, A_{c}, c \in E_{0}\right\rangle$.

Proof. For any $\sigma \in \Gamma_{f} \backslash\left\langle K_{f}, A_{c}, c \in E_{0}\right\rangle$. Applying Lemma 10, $\sigma 0$ is again a cusp. Hence $\sigma 0$ must be $\left\langle K_{f}, A_{c}, c \in E_{0}\right\rangle$-equivalent to 0 . Choose $\delta \in\left\langle K_{f}, A_{c}, c \in E_{0}\right\rangle$ such that $\delta \sigma 0=0$. Then $\delta \sigma \in\left(\Gamma_{f}\right)_{0}$. Hence $\Gamma_{f}=\left\langle K_{f}, B, A_{c}, c \in E_{0}\right\rangle$ holds.

Theorem 13. (Helling's Theorem [5]) The maximal discrete groups of $P S L(2, \mathbb{C})$ commensurable with the modular group $S L(2, \mathbb{Z})$ are just the images of the conjugates of $\Gamma_{0}(N)+$ for square free $N$.

Corollary 14. For each irreducible character $\chi$ of $\mathbb{M}$, the set of prime divisors of the index $\left[\Gamma_{\chi}: \Gamma_{0}\left(N_{\chi}\right)\right]$ is a subset of $\{2,3,5,7\}$.

Proof. By Helling's Theorem, any maximal subgroup that contains $\Gamma_{\chi}$ is a conjugate of some $\Gamma_{0}(n)+$. Conway has shown in [1] that $n$ must be a divisor of $N_{\chi}$. Now compare the volumes of the fundamental domains of $S L_{2}(\mathbb{Z}), \Gamma_{0}(n)$, and $\Gamma_{0}\left(N_{\chi}\right)$. Noting that the conjugation does not change the volume and that the normalizer of $\Gamma_{0}(n)$ changes the volume of the fundamental domain by a factor involving only primes 2 and 3 , we obtain our lemma since the index $\left[S L_{2}(\mathbb{Z})\right.$ : $\left.\Gamma_{\nvdash}\left(\mathbb{N}_{\chi}\right)\right]$ involves only primes $2,3,5$, and 7 .

Let $\chi$ be an irreducible character of $\mathbb{M}$ and $\Gamma_{\chi}$ be the invariance group of $t_{\chi}(z)$. We are now ready to prove :

(1). $A_{0}=\emptyset$, and,

(2). $\left(\Gamma_{\chi}\right)_{0}=\left(\Gamma_{0}\left(N_{\chi}\right)\right)_{0}$.

Lemma 15. Let $\chi$ be an irreducible character of $\mathbb{M}$ and let $c$ be a cusp of $\Gamma_{0}\left(N_{\chi}\right)$, not equivalent to 0 . Then $A_{0}=\left\{A_{c} \mid A_{c} 0=c\right\}=\emptyset$. 
Proof. We first recall that the singular part of $\frac{1}{|\mathbb{M}|} \sum_{g \in \Phi_{c}} \chi(g)\left(t_{g} \mid P_{c}\right)(z)$ at $z=$ $\infty i$ is given by

$$
\operatorname{sing}_{P_{c}} t_{\chi}=\frac{1}{|\mathbb{M}|} \sum_{g \in \Phi_{c}} \chi(g) \frac{\sigma_{g}}{e^{2 \pi i U_{c, g} z}} .
$$

Applying Lemmas 7, 9 and 10 and Corollary 8, we see that it suffices to show that $\operatorname{sing}_{P_{0}} t_{\chi}$ can not be derived from $\operatorname{sing}_{P_{c}} t_{\chi}$ under the substitution $z \rightarrow a z+b$ if $c \neq 0$. This is achieved by a case-by-case study. We give an example to indicate how the lemma is proved.

Example 3. Let $\chi$ be the trivial character of $\mathbb{M}$. Then $A_{0}=\emptyset$.

Proof. We first note that for any irreducible character $\chi$ of $\mathbb{M}$ and $c \in \mathbb{Q} \cup\{\infty\}$, we have :

(1). The lowest terms in $\operatorname{sing}_{P_{c}} t_{\chi}$ and $\operatorname{sing}_{P_{0}} t_{\chi}$ are of the form $\frac{r}{q}$ for some number $r \in \mathbb{Q}$, and

(2). Terms in $\operatorname{sing}_{P_{c}} t_{\chi}$ and $\operatorname{sing}_{P_{0}} t_{\chi}$ are all of the form $\frac{r}{q^{\frac{1}{t}}}$ (Corollary 8 , Lemma 9 ), for some $r \in \mathbb{Q}$, and $t \in \mathbb{N}$.

Since the lowest term in $\operatorname{sing}_{P_{0}} t_{\chi}$ is $\frac{r}{q}, r \neq 0$, the transformation that sends $\operatorname{sing}_{P_{c}} t_{\chi}$ to $\operatorname{sing}_{P_{0}} t_{\chi}$ is of the form $z \rightarrow a z+b$ where $a$ is some positive integer.

Let $\chi$ is the trivial character, then by Corollary 8 and Lemma 9, one has

$$
\operatorname{sing}_{P_{0}} t_{\chi}=\frac{1}{|\mathbb{M}|} \sum_{g \in \Phi_{0}} \frac{\sigma_{g}}{q^{\frac{1}{n^{h_{g}}}}}=\frac{1}{|\mathbb{M}|}\left(\frac{1}{q}+\frac{2|\mathbb{M}|}{\left|\mathbb{C}_{\mathbb{M}}(\not \nVdash \mathbb{A})\right|} \frac{\sigma_{71 A}}{q^{\frac{1}{71}}}+\cdots\right),
$$

and for any cusp $c=\frac{x}{y}, \operatorname{gcd}(x, y)=1$,

$$
\operatorname{sing}_{P_{c}} t_{\chi}=\frac{1}{|\mathbb{M}|} \sum_{g \in \Phi_{c}} \frac{\sigma_{g}}{e^{2 \pi i U_{c, g} z}}
$$

Suppose $\operatorname{sing}_{P_{0}} t_{\chi}$ can be derived from $\operatorname{sing}_{P_{c}} t_{\chi}$ under $z \rightarrow a z+b$.

(3). We will show $\operatorname{gcd}(y, 71)=1$. Suppose false. Then $71 \mid y$ and $71 A, 71 B \in \Phi_{c}$. Since $\frac{1}{q^{\frac{1}{71}}}$ appears in $\operatorname{sing}_{P_{0}} t_{\chi}$, there exists, by Lemma 11 , some $g$ in $\Phi_{c}$ such that

$$
\frac{\sigma_{g}}{e^{2 \pi i U_{c, g} z}} \mid(z \rightarrow a z+b)=\frac{r}{q^{\frac{1}{71}}}
$$

where $r$ is some constant. Hence

$$
\frac{\operatorname{gcd}\left(h_{g}, y\right)^{2} a}{e_{g} h_{g}^{2}}=\frac{1}{71}
$$

where

$$
e_{g}=\frac{\frac{n_{g}}{h_{g}}}{\operatorname{gcd}\left(\frac{n_{g}}{h_{g}}, \frac{y}{\operatorname{gcd}\left(y, h_{g}\right)}\right)} .
$$

Since $a$ is an integer, this implies that $g=71 A$ or $71 B$. Since $\Gamma_{71 A}=\Gamma_{71 B}=71+$, we have $n_{71 A}=n_{71 B}=71, h_{71 A}=h_{71 B}=1, e_{71 A}=e_{71 B}=71$, and $a=1$. On the other hand, Corollary 8 implies $t_{g} \mid P_{c}=\sigma_{g} t_{g}\left(z-u_{g}\right)$ for $g=71 A$ or $71 B$. Hence the transformation $(*)$ can not be done. This forces

$$
\operatorname{gcd}(y, 71)=1 \text {. }
$$


(4). Since $\frac{1}{q^{\frac{1}{t}}}, t \in\{29,41,59,92,93,94,95,104,110,119\}$ all appear with nonzero coefficients in $\operatorname{sing} P_{0} t_{\chi}$, we may similarly conclude that $\operatorname{gcd}(y, p)=1$ for the other prime divisors of $N_{0}$.

Hence $\operatorname{gcd}\left(y, N_{0}\right)=1$ and $c$ is equivalent to 0 . Consequently, $A_{0}=\emptyset$.

Lemma 16. $\left(\Gamma_{\chi}\right)_{0}=\left(\Gamma_{0}\left(N_{\chi}\right)_{0}\right.$.

Proof. Suppose not. Applying Corollary 14, we see that $\left(\Gamma_{\chi}\right)_{0}$ contains $B_{r}=$ $\left(\begin{array}{ll}1 & 0 \\ \frac{N_{\chi}}{r} & 1\end{array}\right)$ for $r=2,3,5$ or 7 . This implies that the cusp $\infty$ is equivalent to $\frac{r}{N_{\chi}}$ in $\Gamma_{\chi}^{r}$. Therefore $\operatorname{sing} g_{\infty} t_{\chi}$ must be derived from $\operatorname{sing}_{P_{\frac{r}{N_{\chi}}}} t_{\chi}$ under the substitution $z \rightarrow a z+b$. We can now apply an analogous procedure (using $y=\frac{r}{N_{\chi}}$ ) as in Example 3 to get a contradiction.

Remark. One can also prove Lemma 16 by claiming that $B_{r}$ does not leave $t_{\chi}(z)$ invariant. Note that it is easy to show the claim since $B_{r}$ leaves most of the $t_{g}(z)$ invariant except for those $g$ 's such that $n_{g} h_{g}$ is not a divisor of $\frac{N_{\chi}}{r}$.

Combining Lemma 15 and 16, we have :

Theorem 17. Let $\chi$ be an irreducible character of $\mathbb{M}$. Then $\Gamma_{\chi}=\Gamma_{0}\left(N_{\chi}\right)$.

$N_{\chi}$ can be found in Table 1.

Remark. (1). In Lemma 15 and 16, 0 is a better choice than the other cusps $\left(\infty\right.$, for example) since among all the $\operatorname{sing}_{P_{c}} t_{\chi}$ 's, $\operatorname{sing}_{P_{0}} t_{\chi}$ is the one that involves most nonzero terms.

(2). $N_{\chi}$ and its prime decomposition is calculated by a software called GAP. 


\begin{tabular}{|c|c|c|}
\hline$\chi_{i}$ & $N_{\chi_{i}}$ & $\begin{array}{l}\text { Table } 1 \\
N_{\chi_{i}}(\text { prime decomposition })\end{array}$ \\
\hline 1 & 2331309585756753201600 & $2^{6} 3^{3} 5^{2} 7.11 .13 .17 \cdot 19 \cdot 23 \cdot 29.31 .41 .47 .59 .71$ \\
\hline 2 & 11841091337275200 & $2^{6} 3^{3} 5^{2} 7.11 .13 .17 .19 .23 .29 .31 .41$ \\
\hline 3 & 437868837806400 & $2^{6} 3^{3} 5^{2} 7.11 .13 .17 .19 .23 .29 .47$ \\
\hline 4 & 467584848090400 & $2^{5} 3^{3} 5^{2} 7.11 .13 .17 .19 .23 .41 .71$ \\
\hline 5 & 38732026132800 & $2^{6} 3^{3} 5^{2} 7.11 .13 .17 .19 .47 .59$ \\
\hline 6 & 20350725595200 & $2^{6} 3^{3} 5^{2} 7.11 .13 .17 .19 .31 .47$ \\
\hline 7 & 87358471200 & $2^{5} 3^{2} 5^{2} 7.11 .13 .17 .23 .31$ \\
\hline 8 & 7820482269600 & $2^{5} 3^{2} 5^{2} 7.11 .13 .17 .29 .31 .71$ \\
\hline 9 & 18526958049600 & $2^{6} 3^{2} 5^{2} 7.11 .13 .23 .29 .41 .47$ \\
\hline 10 & 222987885120 & $2^{6} 3^{3} 5.7 .11 .13 .19 .23 .59$ \\
\hline 11 & 8490081600 & $2^{6} 3^{2} 5^{2} 7.11 .13 .19 .31$ \\
\hline 12 & 19445025600 & $2^{6} 3^{2} 5^{2} 7.11 .13 .19 .71$ \\
\hline 13 & 9958865716800 & $2^{6} 3^{3} 5^{2} 7.11 .13 .17 .19 .23 .31$ \\
\hline 14 & 73513400 & $2^{5} 3^{3} 5.7 .11 .13 .17$ \\
\hline 15 & 2244077793757800 & $2^{3} 3^{3} 5^{2} 7.11 .13 .17 .19 .23 .29 .41 .47$ \\
\hline 16 & 3749442460305984 & $2^{6} 3^{3} 13.23 .29 .31 .41 .47 .59 .71$ \\
\hline 17 & 3749442460305984 & $2^{6} 3^{3} 13.23 .29 .31 .41 .47 .59 .71$ \\
\hline 18 & 726818400 & $2^{5} 3^{3} 5^{2} 7.11 .19 .23$ \\
\hline 19 & 9182927033280 & $2^{6} 3^{3} 5.7 .11 .13 .19 .29 .41 .47$ \\
\hline 20 & 35703027360 & $2^{5} 3^{2} 5.7 .11 .13 .17 .31 .47$ \\
\hline 21 & 98066928960 & $2^{6} 3^{3} 5.7 .11 .13 .17 .23 .29$ \\
\hline 22 & 22789166400 & $2^{6} 3^{3} 5^{2} 7.11 .13 .17 .31$ \\
\hline 23 & 451392480 & $2^{5} 3^{3} 5.7 .11 .23 .59$ \\
\hline 24 & 295495200 & $2^{5} 3^{2} 5^{2} 7.11 .13 .41$ \\
\hline 25 & 253955520 & $2^{6} 3^{3} 5.7 .13 .17 .19$ \\
\hline 26 & 479256378753600 & $2^{6} 3^{2} 5^{2} 7.19 .31 .41 .47 .59 .71$ \\
\hline 27 & 479256378753600 & $2^{6} 3^{2} 5^{2} 7.19 .31 .41 .47 .59 .71$ \\
\hline 28 & 27003936960 & $2^{6} 3^{2} 5.7 .11 .13 .17 .19 .29$ \\
\hline 29 & 81995760 & $2^{4} 3^{3} 5.7 .11 .17 .29$ \\
\hline 30 & 69618669120 & $2^{6} 3^{2} 5.7 .11 .13 .19 .31 .41$ \\
\hline 31 & 21416915520 & $2^{6} 3^{2} 5.7 .11 .13 .17 .19 .23$ \\
\hline 32 & 214885440 & $2^{6} 3^{3} 5.7 .11 .17 .19$ \\
\hline 33 & 2882880 & $2^{6} 3^{2} 5.7 .11 .13$ \\
\hline 34 & 332640 & $2^{5} 3^{3} 5.7 .11$ \\
\hline 35 & 786240 & $2^{6} 3^{3} 5.7 .13$ \\
\hline 36 & 11147099040 & $2^{5} 3^{3} 5.7 .11 .23 .31 .47$ \\
\hline 37 & 331962190560 & $2^{5} 3^{2} 5.7 .11 .13 .17 .19 .23 .31$ \\
\hline 38 & 333637920 & $2^{5} 3^{3} 5.7 .11 .17 .59$ \\
\hline 39 & 845013600 & $2^{5} 3^{3} 5^{2} 19.29 .71$ \\
\hline 40 & 845013600 & $2^{5} 3^{3} 5^{2} 19.29 .71$ \\
\hline 41 & 16676856385200 & $2^{4} 3^{3} 5^{2} 11.23 .31 .47 .59 .71$ \\
\hline 42 & 16676856385200 & $2^{4} 3^{3} 5^{2} 11.23 .31 .47 .59 .71$ \\
\hline 43 & 186902100 & $2^{2} 3^{3} 5^{2} 7.11 .29 .31$ \\
\hline 44 & 46955594400 & $2^{5} 3.5^{2} 11.13 .41 .47 .71$ \\
\hline
\end{tabular}




\begin{tabular}{|c|c|c|}
\hline 45 & 46955594400 & $2^{5} 3.5^{2} 11.13 .41 .47 .71$ \\
\hline 46 & 54880846020 & $2^{2} 3^{2} 5.7 .11 .13 .17 .19 .23 .41$ \\
\hline 47 & 105386400 & $2^{5} 3^{3} 5^{2} 7.17 .41$ \\
\hline 48 & 105386400 & $2^{5} 3^{3} 5^{2} 7.17 .41$ \\
\hline 49 & 49584815280 & $2^{4} 3^{3} 5.7 .11 .13 .17 .19 .71$ \\
\hline 50 & 6404580 & $2^{2} 3^{2} 5.7 .13 .17 .23$ \\
\hline 51 & 12916800 & $2^{6} 3^{3} 5^{2} 13.23$ \\
\hline 52 & 646027200 & $2^{6} 3^{2} 5^{2} 7.13 .17 .29$ \\
\hline 53 & 228731328 & $2^{6} 3^{2} 7.17 .47 .71$ \\
\hline 54 & 228731328 & $2^{6} 3^{2} 7.17 .47 .71$ \\
\hline 55 & 19044013248 & $2^{6} 3^{3} 13.23 .29 .31 .41$ \\
\hline 56 & 10944013248 & $2^{6} 3^{3} 13.23 .29 .31 .41$ \\
\hline 57 & 25077360 & $2^{4} 3.5 .7 .11 .23 .59$ \\
\hline 58 & 198918720 & $2^{6} 3^{3} 5.7 .11 .13 .23$ \\
\hline 59 & 19433872080 & $2^{4} 3^{3} 5.7 .23 .29 .41 .47$ \\
\hline 60 & 19433872080 & $2^{4} 3^{3} 5.7 .23 .29 .41 .47$ \\
\hline 61 & 2784600 & $2^{3} 3^{2} 5^{2} 7.13 .17$ \\
\hline 62 & 245044800 & $2^{6} 3^{2} 5^{2} 7.11 .13 .17$ \\
\hline 63 & 57266969760 & $2^{5} 3^{3} 5.7 .11 .13 .17 .19 .41$ \\
\hline 64 & 157477320 & $2^{3} 3^{2} 5.7 .11 .13 .19 .23$ \\
\hline 65 & 818809200 & $2^{4} 3^{2} 5^{2} 11.23 .29 .31$ \\
\hline 66 & 263877213600 & $2^{5} 3^{2} 5^{2} 7.11 .13 .19 .41 .47$ \\
\hline 67 & 1588466880 & $2^{6} 3^{2} 5.7 .11 .13 .19 .29$ \\
\hline 68 & 33005280 & $2^{5} 3.5 .7 .11 .19 .47$ \\
\hline 69 & 937440 & $2^{5} 3^{3} 5.7 .31$ \\
\hline 70 & 32864832 & $2^{6} 3^{3} 7.11 .13 .19$ \\
\hline 71 & 182584514112 & $2^{6} 3.7 .11 .13 .17 .29 .41 .47$ \\
\hline 72 & 182584514112 & $2^{6} 3.7 .11 .13 .17 .29 .41 .47$ \\
\hline 73 & 982080 & $2^{6} 3^{2} 5.11 .31$ \\
\hline 74 & 33542208 & $2^{6} 3^{3} 7.47 .59$ \\
\hline 75 & 33542208 & $2^{6} 3^{3} 7.47 .59$ \\
\hline 76 & 7650720 & $2^{5} 3^{3} 5.7 .11 .23$ \\
\hline 77 & 931170240 & $2^{6} 3^{2} 5.7 .11 .13 .17 .19$ \\
\hline 78 & 33754921200 & $2^{4} 3^{2} 5^{2} 7.11 .13 .17 .19 .29$ \\
\hline 79 & 42325920 & $2^{5} 3^{2} 5.7 .13 .17 .19$ \\
\hline 80 & 4969440 & $2^{5} 3^{2} 5.7 .17 .29$ \\
\hline 81 & 63126554400 & $2^{5} 3^{2} 5^{2} 7.13 .23 .59 .71$ \\
\hline 82 & 63126554400 & $2^{5} 3^{2} 5^{2} 7.13 .23 .59 .71$ \\
\hline 83 & 208304928 & $2^{5} 3^{2} 13.23 .41 .59$ \\
\hline 84 & 208304928 & $2^{5} 3^{2} 13.23 .41 .59$ \\
\hline 85 & 704223936 & $2^{6} 3^{3} 13.23 .29 .47$ \\
\hline 86 & 704223936 & $2^{6} 3^{3} 13.23 .29 .47$ \\
\hline 87 & 1235520 & $2^{6} 3^{3} 5.11 .13$ \\
\hline 88 & 3967200 & $2^{5} 3^{2} 5^{2} 19.29$ \\
\hline 89 & 11737440 & $2^{5} 3^{3} 5.11 .13 .19$ \\
\hline 90 & 11737440 & $2^{5} 3^{3} 5.11 .13 .19$ \\
\hline 91 & 2542811040 & $2^{5} 3^{2} 5.7 .11 .17 .19 .71$ \\
\hline 92 & 22102080 & $2^{6} 3.5 .7 .11 .13 .23$ \\
\hline 93 & 1441440 & $2^{5} 3^{2} 5.7 .11 .13$ \\
\hline 94 & 879840 & $2^{5} 3^{2} 5.13 .47$ \\
\hline
\end{tabular}




\begin{tabular}{|c|c|c|}
\hline 95 & 16576560 & $2^{4} 3^{2} 5.7 .11 .13 .23$ \\
\hline 96 & 21677040 & $2^{4} 3^{2} 5.7 .11 .17 .23$ \\
\hline 97 & 5267201940 & $2^{2} 3^{2} 5.7 .11 .13 .23 .31 .41$ \\
\hline 98 & 7900200 & $2^{3} 3^{3} 5^{2} 7.11 .19$ \\
\hline 99 & 1660401600 & $2^{6} 3.5^{2} 11.13 .41 .59$ \\
\hline 100 & 1660401600 & $2^{6} 3.5^{2} 11.13 .41 .59$ \\
\hline 101 & 932769600 & $2^{6} 3.5^{2} 7.17 .23 .71$ \\
\hline 102 & 7601451872175 & $3^{3} 5^{2} 7.17 .19 .29 .41 .59 .71$ \\
\hline 103 & 7601451872175 & $3^{3} 5^{2} 7.17 .19 .29 .41 .59 .71$ \\
\hline 104 & 6511680 & $2^{6} 3^{2} 5.7 .17 .19$ \\
\hline 105 & 5844589984800 & $2^{5} 3^{2} 5^{2} 7.19 .31 .47 .59 .71$ \\
\hline 106 & 5844589984800 & $2^{5} 3^{2} 5^{2} 7.19 .31 .47 .59 .71$ \\
\hline 107 & 2434219200 & $2^{6} 3^{2} 5^{2} 7.19 .31 .41$ \\
\hline 108 & 2434219200 & $2^{6} 3^{2} 5^{2} 7.19 .31 .41$ \\
\hline 109 & 280800 & $2^{5} 3^{3} 5^{2} 13$ \\
\hline 110 & 947520 & $2^{6} 3^{2} 5.7 .47$ \\
\hline 111 & 1016747424 & $2^{5} 3^{3} 7.11 .17 .29 .31$ \\
\hline 112 & 386100 & $2^{2} 3^{3} 5^{2} 11.13$ \\
\hline 113 & 6568800 & $2^{5} 3.5^{2} 7.17 .23$ \\
\hline 114 & 1374912 & $2^{6} 3^{2} 7.11 .31$ \\
\hline 115 & 151200 & $2^{5} 3^{3} 5^{2} 7$ \\
\hline 116 & 92400 & $2^{4} 3.5^{2} 7.11$ \\
\hline 117 & 411840 & $2^{6} 3^{2} 5.11 .13$ \\
\hline 118 & 19562400 & $2^{5} 3^{2} 5^{2} 11.13 .19$ \\
\hline 119 & 12524852340 & $2^{2} 3^{3} 5.7 .11 .13 .17 .29 .47$ \\
\hline 120 & 41801760 & $2^{5} 3^{2} 5.7 .11 .13 .29$ \\
\hline 121 & 75698280 & $2^{3} 3^{3} 5.7 .17 .19 .31$ \\
\hline 122 & 8148853440 & $2^{6} 3^{2} 5.7 .13 .17 .31 .59$ \\
\hline 123 & 864175548600 & $2^{3} 3^{3} 5^{2} 7.13 .17 .31 .47 .71$ \\
\hline 124 & 3456702194400 & $2^{5} 3^{3} 5^{2} 7.13 .17 .31 .47 .71$ \\
\hline 125 & 3456702194400 & $2^{5} 3^{3} 5^{2} 7.13 .17 .31 .47 .71$ \\
\hline 126 & 119700 & $2^{2} 3^{2} 5^{2} 7.19$ \\
\hline 127 & 13695552 & $2^{6} 3^{2} 13.31 .59$ \\
\hline 128 & 752016096 & $2^{5} 3^{3} 13.23 .41 .71$ \\
\hline 129 & 752016096 & $2^{5} 3^{3} 13.23 .41 .71$ \\
\hline 130 & 19320840 & $2^{3} 3^{2} 5.7 .11 .17 .41$ \\
\hline 131 & 1004683680 & $2^{5} 3^{2} 5.7 .11 .13 .17 .41$ \\
\hline 132 & 164160 & $2^{6} 3^{3} 5.19$ \\
\hline 133 & 14379596431200 & $2^{5} 3^{3} 5^{2} 7.11 .13 .17 .19 .29 .71$ \\
\hline 134 & 14208480 & $2^{5} 3^{3} 5.11 .13 .23$ \\
\hline 135 & 497653200 & $2^{4} 3^{3} 5^{2} 11.59 .71$ \\
\hline 136 & 497653200 & $2^{4} 3^{3} 5^{2} 11.59 .71$ \\
\hline 137 & 995276700 & $2^{2} 3^{3} 5^{2} 11.23 .31 .47$ \\
\hline 138 & 5109369408 & $2^{6} 3^{3} 11.13 .23 .29 .31$ \\
\hline 139 & 514080 & $2^{5} 3^{3} 5.7 .17$ \\
\hline 140 & 59017104226080 & $2^{5} 3^{3} 5.7 .11 .13 .17 .19 .29 .31 .47$ \\
\hline 141 & 1151710560 & $2^{5} 3^{2} 5.7 .11 .13 .17 .47$ \\
\hline 142 & 3767400 & $2^{3} 3^{2} 5^{2} 7.13 .23$ \\
\hline 143 & 7600320 & $2^{6} 3^{2} 5.7 .13 .29$ \\
\hline 144 & 11823840 & $2^{5} 3^{3} 5.7 .17 .23$ \\
\hline
\end{tabular}




\begin{tabular}{|c|c|c|}
\hline 145 & 8558550 & $2.3^{2} 5^{2} 7.11 .13 .19$ \\
\hline 146 & 664020 & $2^{2} 3^{2} 5.7 .17 .31$ \\
\hline 147 & 4320 & $2^{5} 3^{3} 5$ \\
\hline 148 & 655188534 & $2.3^{3} 7.11 .13 .17 .23 .31$ \\
\hline 149 & 102240 & $2^{5} 3^{2} 5.71$ \\
\hline 150 & 157248 & $2^{6} 3^{3} 7.13$ \\
\hline 151 & 26489342880 & $2^{5} 3^{2} 5.7 .11 .13 .17 .23 .47$ \\
\hline 152 & 93276960 & $2^{5} 3.5 .7 .17 .23 .71$ \\
\hline 153 & 13137600 & $2^{6} 3.5^{2} 7.17 .23$ \\
\hline 154 & 428400 & $2^{4} 3^{2} 5^{2} 7.17$ \\
\hline 155 & 18221280 & $2^{5} 3.5 .7 .11 .17 .29$ \\
\hline 156 & 190072512 & $2^{6} 3^{2} 7.17 .47 .59$ \\
\hline 157 & 21176100 & $2^{2} 3^{3} 5^{2} 11.23 .31$ \\
\hline 158 & 37130940 & $2^{2} 3^{3} 5.7 .11 .19 .47$ \\
\hline 159 & 852390 & $2.3^{3} 5.7 .11 .41$ \\
\hline 160 & 184363200 & $2^{6} 3^{2} 5^{2} 7.31 .59$ \\
\hline 161 & 108803771818560 & $2^{6} 3^{2} 5.7 .13 .17 .19 .23 .29 .41 .47$ \\
\hline 162 & 1657656 & $2^{3} 3^{2} 7.11 .13 .23$ \\
\hline 163 & 345290400 & $2^{5} 3^{2} 5^{2} 7.13 .17 .31$ \\
\hline 164 & 90014400 & $2^{6} 3^{2} 5^{2} 7.19 .47$ \\
\hline 165 & 30240 & $2^{5} 3^{3} 5.7$ \\
\hline 166 & 4032 & $2^{6} 3^{2} 7$ \\
\hline 167 & 4062240 & $2^{5} 3^{2} 5.7 .13 .31$ \\
\hline 168 & 3204801600 & $2^{6} 3.5^{2} 7.11 .13 .23 .29$ \\
\hline 169 & 24196995900 & $2^{2} 3^{2} 5^{2} 7.11 .17 .19 .23 .47$ \\
\hline 170 & 668304 & $2^{4} 3^{3} 7.13 .17$ \\
\hline 171 & 73920 & $2^{6} 3.5 .7 .11$ \\
\hline 172 & 6983776800 & $2^{5} 3^{3} 5^{2} 7.11 .13 .17 .19$ \\
\hline 173 & 32959080 & $2^{3} 3^{2} 5.7 .11 .29 .41$ \\
\hline 174 & 3115200 & $2^{6} 3.5^{2} 11.59$ \\
\hline 175 & 48163383908640 & $2^{5} 3^{2} 5.7 .11 .13 .17 .19 .31 .47 .71$ \\
\hline 176 & 427211200 & $2^{6} 5^{2} 13.19 .23 .47$ \\
\hline 177 & 858816 & $2^{6} 3^{3} 7.71$ \\
\hline 178 & 21416915520 & $2^{6} 3^{2} 5.7 .11 .13 .17 .19 .23$ \\
\hline 179 & 14400 & $2^{6} 3^{2} 5^{2}$ \\
\hline 180 & 14400 & $2^{6} 3^{2} 5^{2}$ \\
\hline 181 & 154881891350 & $2.3^{3} 5^{2} 11.13 .19 .29 .31 .47$ \\
\hline 182 & 2009280 & $2^{6} 3.5 .7 .13 .23$ \\
\hline 183 & 6339168 & $2^{5} 3^{3} 11.23 .29$ \\
\hline 184 & 26429760 & $2^{6} 3^{3} 5.7 .19 .23$ \\
\hline 185 & 32730048 & $2^{6} 3^{3} 13.31 .47$ \\
\hline 186 & 7425600 & $2^{6} 3.5^{2} 7.13 .17$ \\
\hline 187 & 8237275200 & $2^{6} 3^{2} 5^{2} 7.11 .17 .19 .23$ \\
\hline 188 & 15120 & $2^{4} 3^{3} 5.7$ \\
\hline 189 & 54774720 & $2^{6} 3^{2} 5.7 .11 .13 .19$ \\
\hline 190 & 27989280 & $2^{5} 3^{3} 5.11 .19 .31$ \\
\hline 191 & 34272 & $2^{5} 3^{2} 7.17$ \\
\hline 192 & 3500640 & $2^{5} 3^{2} 5.11 .13 .17$ \\
\hline 193 & 1049200425 & $3^{3} 5^{2} 7.13 .19 .29 .31$ \\
\hline 194 & 1404480 & $2^{6} 3.5 .7 .11 .19$ \\
\hline
\end{tabular}




\section{REFERENCES}

[1] J. H. Conway, Understanding Groups like $\Gamma_{0}(N)$, (preprint).

[2] J. H. Conway and S. P. Norton, Monstrous Moonshine, Bull. London Math. Soc. 11 (1979), 308-338.

[3] I. Frenkel, J. Lepowsky and A. Meurman, Vertex Operator Algebras and the Monster, Academic Press, Inc. (1988).

[4] K. Harada, Modular Functions, Modular Forms and Finite Group, Lecture Notes at The Ohio State University, (1987).

[5] H. Helling, On the Commensurablility Classes of Rational Modular Group, J. London Math. Soc. 2 (1970), 67-72.

[6] J. McKay and H. Strauss, The q-series of Monstrous Moonshine and the Decomposition of the Head Characters, Comm. Alg. 18(1) (1990), 253-278.

DEPARTMENT OF MATHEMATICS, THE OHIO STATE UNIVERSITY, COLUMBUS, OHIO 43210, USA

E-mail address : haradako@math.ohio-state.edu

DEPARTMENT OF MATHEMATICS, NITIONAL UNIVERSITY OF SINGAPORE, SINGAPORE 0511, REPUBLIC OF SINGAPORE

E-mail address : matlml@leonis.nus.sg 